\title{
O PROCESSO DE TERRITORIALIZAÇÃO E A ATENÇÃO À SAÚDE NO PROGRAMA SAÚDE DA FAMÍLIA
}

\author{
THE PROCESS OF BROADENING HEALTH UNIT SCOPE \\ AND HEALTH CARE IN THE FAMILY HEALTH PROGRAM CONTEX \\ EL PROCESO DE TERRITORIALIZACIÓN Y LA ATENCIÓN \\ A LA SALUD EN EL PROGRAMA SALUD DE LA FAMILIA
}

Melissa dos Reis P. Mafra ${ }^{1}$ Maria Marta Nolasco Chaves ${ }^{2}$

Enfermeira do Município de Piraquara-PR.

Mestre em Enfermagem. Professora Assistente do Departamento de Enfermagem da Universidade Federal do Paraná. Área de Saúde Coletiva.

RESUMO: Considerando que o trabalho junto ao Programa Saúde da Família (PSF) deve levar em conta, em primeiro lugar, o conhecimento do território onde se vai atuar, o que significa ir além dos muros da Unidade Básica de Saúde (UBS) e que é fundamental conhecer o território que constitui a área de abrangência da Unidade de Saúde (US) para identificar como vivem, adoecem e morrem as pessoas, desenvolvemos o presente estudo com o objetivo de compreender o processo de territorialização e analisar as mudanças na assistência à saúde em uma Unidade de Saúde do município de Curitiba-PR no período de 2000 a agosto 2004. Para coleta de dados, utilizamos como instrumento a entrevista semi-estruturada e a observação estruturada. A entrevista foi desenvolvida com onze sujeitos que aceitaram participar assinando o Termo de Consentimento Livre e Esclarecido, onde se garantiu o direito ao sigilo, o anonimato e o direito a desistência. Na análise dos dados percebemos que o processo de territorialização ocorreu na U.S., mas nem todos os sujeitos da pesquisa têm a clareza de que a contínua construção do processo deste é a base da discussão e da construção do modelo de assistência à saúde para aquela comunidade. O modelo de assistência prestado pela equipe ainda tem como foco a demanda espontânea no serviço, utilizando a visita domiciliar para prestar atendimento aos portadores de algum adoecimento ou com riscos à saúde identificados. Referem que as mudanças encontradas no período se relacionam ao serviço e que esse é um processo muito lento, sendo afetado pela falta de profissionais e pequeno tempo de permanência destes no serviço local, fazendo-nos refletir a respeito da qualificação dos profissionais para o desenvolvimento do Programa Saúde da Família e que estes necessitariam de uma maior vinculação no serviço para a construção e consolidação do novo modelo de atenção à saúde. Acreditamos que as mudanças que ocorreram no período do início da implantação do serviço tiveram como principal contribuinte a atuação dos profissionais com apoio da comunidade local. Concluímos que este estudo nos fez perceber que estamos longe de desenvolver o modelo de atenção à saúde proposto pelo Programa Saúde da Família. Para tal necessitaremos de muitas investigações, que como esta nos faça refletir sobre as mais diversas realidades de saúde e as ações que os serviços estão desenvolvendo.

PALAVRAS-CHAVE: Saúde da família; Sistema local de Saúde; Planejamento em saúde; Programa saúde da família.

ABSTRACT: Considering that the work performed by the Family Health Program must ultimately take into account the knowledge of the surroundings where it will be effected, which means to go beyond the walls of Primary Health Unit in order to get to know the scope area of the Health Unit as well as identify how those people live, get ill and die, we carried out this study objectifying to understand the process of broadening health units' scope and analyze the changes in health care at Health Unit in Curitiba city - Brazil from 2000 to August, 2004. A semi-structured interview was the instrument used as well as structured observation for data collection. The interviews were carried out with 11 (eleven) subjects who signed a consent form where their rights of secrecy, anonymity and acquittance have been ensured. Analyzing the data, it was evidenced the process of broadening the health unit scope, however not all subjects perceived it as the framework for discussion and model design of health care in that community. The frame of health assistance has still been focused on the spontaneous demand, performing home visits to assist bearers of some kind of disease or diagnosed health risks. It was reported that the changes evidenced along the period are related to the health care and that is a slow process, affected by shortage of professionals and high staff turnover. That has made us think about professionals qualification in order to carry out the Family Health Program, and such professionals would demand a longer stay in the unit so that the new design for health care was on settled and consolidated. We believe that the changes occurred along the initial period of the program were ultimately brought about by the professionals' performance supported by the local community. We concluded that this study made us realize that we are far from undertaking the health care sistem proposed by the Family Health Program. Thus, we deem necessary to carry out many investigations like this in order to think over the several "realities" in the health realm and actions that health services have been performing.

KEYWORDS: Family health strategy; Local health system; Health planning; Family health program.

RESUMEN: Considerando que el trabajo junto al Programa Salud de la Familia (PSF) debe llevar en cuenta en primer plano, el conocimiento del territorio donde se va actuar, lo que significa ir más allá de los muros de la Unidad Básica de Salud (UBS) y que es fundamental conocer el territorio que constituye el área abarcada por la Unidad de Salud (US) para que se pueda identificar como viven, se quedan enfermos y mueren las personas, hemos desarrollado la presente pesquisa que ha tenido como objetivo comprender el proceso de territorialización, es decir, conocer la realidad sociocultural del área abarcada por el Puesto de Salud (PS) y analizar los cambios en la asistencia a la salud en una Unidad de Salud del municipio de Curitiba-Paraná en el periodo de 2000 a agosto de 2004. Para la recolección de datos fue utilizado como instrumento la entrevista semiestructurada y la observación estructurada. La entrevista ha sido desarrollada con once sujetos que aceptaron participar firmando el Término de Consentimiento Libre y Esclarecido donde se ha garantizado el derecho al sigilo, el anonimato, así como, a la desistencia. En el análisis de los datos percibimos que el proceso de territorialización ha ocurrido en la U.S., sin embargo ni todos los sujetos de la pesquisa tienen la claridad de que la construcción continua del proceso es la base de la discusión y de la construcción del modelo de la asistencia a la salud para aquella comunidad. El modelo de la asistencia usado por el equipo, todavía tiene como la demanda espontánea en el servicio, donde es utilizada la visita domiciliar para prestar atendimiento a los portadores de alguna enfermedad o con riesgos de la salud ya ydentificados. Refieren que los cambios encontrados en los periodos se relacionan al servicio y que ese es un proceso lento, donde es afectado por la falta de profesionales o la permanencia corta de ellos en el servicio local, nos hace reflexionar sobre la calificación de estos para el desarrollo del Programa de Salud de la Familia y una mayor vinculación en el servicio para la construcción y consolidación del modelo nuevo de atención a la salud. Creemos que los cambios ocurridos en el periodo inicial de la implantación del servicio han tenido como contribuyente principal, la actuación de los profesionales y algunos apoyos de la comunidad local.Hemos concluido a través de esa pesquisa que estamos lejos de desarrollar el modelo de atención a la salud propuesto por el Programa Salud de la Familia. Para tal, necesitaremos de muchas investigaciones como esta que nos haga reflexionar sobre las más diversas realidades de salud y las acciones que los servicios están desarrollando.

PALABRAS CLAVES: Salud de la familia; Sistemas local de Salud; Planeamiento en salud; Programa salud de la familia.

Recebido em: 05/11/2003

Aceito em: $06 / 02 / 2004$
Maria Marta Nolasco Chaves

Rua Pe. Camargo, 120 - Curitiba - PR

E-mail: denfer@ufpr.br 


\section{INTRODUÇÃO}

O presente artigo apresenta a investigação sobre o processo de territorialização e as mudanças que ocorreram na assistência à saúde em uma Unidade de Saúde do Município de Curitiba-PR, no período de 2000 a agosto de 2004, com a implantação do Programa de Saúde da Família.

No Brasil, o processo de reflexão e reformulação do Sistema de Saúde consolidou-se na década dos anos 80 com o Sistema Único de Saúde (SUS), e no início da década de 90 foi instituído o Programa de Agentes Comunitários de Saúde (PACS) como uma forma de transição do modelo de atenção à saúde vigente para o modelo que objetiva reestruturar as ações curativas para ações de promoção e proteção da saúde. Em 1994 o Ministério da Saúde, com o objetivo de consolidar o modelo de atenção à saúde proposto, adotou como estratégia política o Programa Saúde da Família (PSF). Assim, o programa passa a ser implementado nos municípios que aderem ao convênio proposto pelo Ministério da Saúde a partir do ano de 1995.

Assim, o PSF é fundamentado em princípios e diretrizes que priorizam o desenvolvimento de ações de promoção, proteção e recuperação da saúde dos indivíduos e da família nos diferentes momentos do ciclo de vida, de forma integral e contínua, por meio das atividades das equipes de saúde, que promovem e desenvolvem o atendimento na Unidade Local de Saúde nos domicílios e na própria comunidade.

O trabalho junto ao PSF deve levar em conta, em primeiro lugar, o conhecimento do território onde se vai atuar, o que significa ir além dos muros da Unidade Básica de Saúde (UBS) ${ }^{1}$. É fundamental conhecer o território que constitui a área de abrangência da Unidade de Saúde (US) para identificar como vivem, adoecem e morrem as pessoas. Assim, esta estratégia de ação em saúde se contrapõe às propostas de criação de programas de atenção à saúde que são aplicados, indistintamente, em todo o território nacional, sem levar em consideração as especificidades da demanda. Este modelo na Saúde
Coletiva foi adotado por muitos anos e não gerou mudanças na assistência à saúde que dessem conta das questões que o setor deveria enfrentar.

Acreditamos que ao levar em consideração as necessidades locais por meio da caracterização da situação epidemiológica, a proposta de intervenção na saúde pode melhorar os níveis de resolubilidade nos atendimentos. Logo, para desenvolvermos uma assistência à saúde mais resolutiva, deveremos identificar os problemas de saúde mais relevantes e prioritários para a comunidade para a partir destes estabelecermos a proposta de intervenção. Esta proposta difere significativamente do modelo que estabelecia atividades idealizadas por poucos, onde se privilegiava a técnica e desconsiderava a realidade onde esta seria desenvolvida.

Segundo Rouquayrol ${ }^{2}$, observa-se uma multiplicidade de enfoques e propostas metodológicas que subsidiam a formulação de políticas, organização e a avaliação de sistemas de saúde, para posterior elaboração de projetos e reorientações para o enfrentamento de problemas atendendo as necessidades da comunidade através de determinações geográficas, demográficas, epidemiológicas e sociais. Assim sendo, para dar conta desta investigação estabelecemos os seguintes objetivos: investigar 0 processo de territorialização para a construção do modelo de assistência à saúde prestada à população da área de abrangência da Unidade de Saúde; compreender o processo de territorialização desenvolvido na área de abrangência da Unidade de Saúde e analisar as mudanças na assistência à saúde na Unidade de Saúde no período de 2000 a agosto de 2004.

\section{METODOLOGIA}

A abordagem metodológica adotada para o desenvolvimento deste trabalho foi de natureza exploratória descritiva, de caráter qualitativo, por meio de observação estruturada no desenvolvimento do trabalho da equipe e coleta de dados em entrevistas 
semi-estruturadas realizadas com profissionais de saúde e liderança local.

O método estruturado da observação, segundo Polit e Hungler ${ }^{3}$, consiste em observar o contexto, e este processo conduz o pesquisador a sua inserção no mesmo, produzindo com isto uma interação social mais ampla. Neste caso, o desenvolvimento deste estudo permitiu ao investigador aproximar-se da prática sanitária no PSF e, assim, possibilitou a reflexão e a experiência com a equipe de saúde local.

Os dados foram coletados no período compreendido entre os meses de outubro a novembro de 2004. Os aspectos éticos e legais respeitaram a Resolução 196/96 do CNS que garante o anonimato, o sigilo e direito de desistir sem prejuízo para os envolvidos e que a proposta desta investigação foi submetida à apreciação do comitê de ética do Setor de Ciências da Saúde da Universidade Federal do Paraná, que aprovou a mesma.

Para a realização das entrevistas durante o período de trabalho na Unidade de Saúde, foi necessário fazer convite individual para possibilitar a participação na pesquisa. Ao aceitarem participar, os sujeitos assinaram o Termo de Consentimento Livre e Esclarecido. As entrevistas foram realizadas no período da manhã, durante o expediente de trabalho, sem que houvesse interferências neste. O tempo de cada entrevista durou, em média, 10 minutos e foram realizadas com onze sujeitos. A repetição de dados, saturação segundo Minayo ${ }^{4}$, foi o critério para interrompermos a coleta de dados e iniciarmos as análises das falas.

\section{ANÁLISE E DISCUSSÃO}

Os profissionais, sujeitos deste estudo, foram caracterizados segundo a faixa etária da seguinte forma: seis entre 41 e 50 anos, seguida de três com idade entre 31 e 40 anos, um sujeito entre 21 e 30 anos e um acima de 51 anos. Com relação à função que ocupam na Unidade de Saúde tivemos: quatro auxiliares de enfermagem, quatro agentes comunitários de saúde; uma enfermeira; uma cirurgiã dentista e uma técnica de higiene dental. Sendo o tempo de atuação no PSF: 6 meses a 2 anos e 6 meses, quatro sujeitos; 3 a 5 anos, quatro; e de 8 a 10 anos, três sujeitos.

Outro dado a ser destacado é o tempo de atuação na Unidade de Saúde, que foi inaugurada há quatro anos e meio, pois verificamos que poucos são os profissionais que estão desde o seu início, sendo que a maioria (cinco sujeitos) está entre o período que compreende de 6 meses até 2 anos, seguido por quatro que estão na U.S. entre 2 e 4 anos e apenas dois dos profissionais estão há mais de 4 anos. Esta diferença de tempo de inserção na referida U.S. poderia ser um fator determinante para a intervenção em saúde na área de abrangência, pois podemos pressupor que os profissionais inseridos por um período mais longo, naquele local, desenvolveriam suas percepções sobre a realidade local e teriam os seus desempenhos mais comprometidos com os problemas de saúde ali existentes.

Brasil (2004), afirma que o pequeno tempo de permanência dos profissionais nas equipes de saúde do PSF dificulta a qualificação destes profissionais e o desempenho das ações, relacionados com a necessidade de adesão e incorporação de novos valores e o exercício de novas práticas de saúde, constituindo-se num aspecto importante para o trabalho na saúde.

Quanto ao processo de territorialização desenvolvido na Unidade de Saúde, a maioria dos entrevistados relatou ter colaborado com o mesmo. Somente dois dos entrevistados referiram não ter participado do processo. Para desenvolver o referido processo, os entrevistados citaram como formas e recursos mais utilizados para a realização da territorialização: o mapeamento, a contagem de pessoas (inquérito ou arrastão) e a (re) divisão da área de abrangência. Cabe destacar que apenas um sujeito referiu que a territorialização é um processo contínuo, que é alimentado com dados coletados diretamente da realidade. 
Pudemos perceber nas falas que os profissionais consideram o território como área geográfica onde se contam pessoas que ali vivem e onde se divide e 're-divide' áreas sem considerar a dinâmica da realidade como essencial para se fundamentar uma proposta de intervenção. No processo de terrritorialização deve-se fazer o mapeamento social antes de realizar o mapeamento geográfico ${ }^{5}$. A autora destaca que esta priorização é para que se possa não só atuar sobre conseqüências, mas também sobre as causas dos problemas de saúde e que a intervenção em saúde não pode se restringir à construção de Unidades de Saúde, mas sim em propostas que desencadeiam ações intersetoriais para promover as transformações na realidade sanitária.

Neste caso, os problemas de saúde no território adstrito foram identificados pelos sujeitos em suas falas como sendo a presença de doenças crônicas (diabetes e hipertensão); saúde mental, dando-se destaque para alcoolismo e drogadição. Houve referência também para problemas estruturais da sociedade que interferem nas condições e qualidade de vida. Neste sentido, ao analisarmos os discursos encontramos questões relacionadas à falta de saneamento, habitação irregular, miséria, fome, desemprego, dificuldade financeira, falta de estrutura familiar e renda familiar como problemas de saúde.

Um dos entrevistados referiu questões culturais como problema de saúde. Porém, apresentou contradição quando na mesma fala destacou a condição econômica vivida pelos moradores como sendo um dos problemas de saúde naquela realidade. Percebemos, neste caso, a dificuldade de compreensão, por parte do sujeito, sobre o que seriam os fatores culturais citados, uma vez que o próprio sujeito destaca a situação financeira e as moradias em áreas de risco como problema de saúde.

Trad et $\mathrm{al}^{6}$, refere que o PSF foi implantado nas áreas onde se concentram as populações mais pobres e com as piores condições de infra-estrutura básica, porém constata-se nestes grupos populacionais um elevado grau de satisfação dos usuários, principalmente com relação às visitas domiciliares. Entretanto, reclamam da falta de atendimento da demanda. A autora refere ainda que o nível de aceitação do serviço está condicionado ao grau de escolaridade e de eficácia da comunicação e educação em saúde prestada pela U.S., sendo os profissionais surpreendidos quando percebem que a lógica das necessidades dos usuários não corresponde à lógica do planejamento e da assistência realizada.

Ao falarem sobre o que acham da intervenção em saúde promovida pela Unidade, os sujeitos referiram que esta se desenvolve por meio de grupos, individualmente ou por visitas domiciliares. Somente um dos sujeitos destacou a participação popular como um subsídio para melhorar o serviço de saúde local.

Quanto à qualificação da intervenção que promovem, alguns dos sujeitos referiram que consideram esta como: boa, muito boa imprescindível. Porém, se contradizem ao afirmarem que esta é realizada na medida do possível ou conforme as possibilidades da Unidade de Saúde.

Neste caso, percebemos a contradição na compreensão do que seria esta "qualidade" da assistência prestada, pois consideram as ações que desenvolvem de boa qualidade, porém dentro das possibilidades do serviço e na medida dos recursos que este dispõe. Assim, ao relacionarem anteriormente as questões de saúde a serem enfrentadas, afirmam que estas estão além das condições dadas para o trabalho que desenvolvem, ou seja, relacionam questões como a falta de saneamento, a habitação irregular, a miséria, a fome, a falta de escolaridade, o desemprego e a falta de estrutura e renda familiar. Portanto, nas questões a serem enfrentadas os sujeitos referiram problemas que vão muito além de questões biológicas e que estas deveriam ser enfrentadas e solucionadas com intervenções intersetoriais e equipes multidisciplinares. Assim, ao afirmarem que as ações desenvolvidas são de boa qualidade, negam as suas percepções sobre os problemas de saúde que afirmaram ser determinados pelas questões econômicas e sociais. Aqui há a negação de que estas questões deveriam ser 
consideradas para uma intervenção mais efetiva na saúde e que estas não serão resolvidas com ações específicas e limitadas pelos recursos do setor.

Para Oliveira ${ }^{7}$, este é o retrato de um modelo de assistência à saúde que desconsidera os aspectos sociais e culturais dos indivíduos, tendo como base para a assistência o cientificismo e o biologicismo e de nada adiantaria aumentar a alocação de recursos para o setor, configurando a definição de Mendes, citado pelo autor, que esta é uma crise paradigmática.

Quanto à identificação de mudanças na realidade local, todos referiram que estas ocorreram mas, nas falas, destacaram que as modificações ocorridas no serviço não referiram alterações significativas nas questões de saúde na área de abrangência. Algumas das mudanças citadas nas questões de saúde foram relacionadas à melhora na conscientização dos pacientes quanto à higiene pessoal e residencial; controle de hipertensos e diabéticos; questões relacionadas aos programas para idosos e a alimentação, implantação dos programas e envolvimento dos agentes comunitários nas atividades com a população local.

A falta de equidade no acesso ao serviço, apoio diagnóstico e referência ainda são insuficientes para garantir a resolubilidade e continuidade de atenção às populações atendidas pelo PSF principalmente no que diz respeito às maiores complexidades, havendo a necessidade de estruturação da rede do SUS e implementação de controle e regulação do sistema ${ }^{8}$.

Um dos sujeitos destacou que as intervenções nas questões de saúde fazem com que estas se desloquem para outras questões que surgirão posteriormente devido a não intervenção nos determinantes dos problemas enfrentados. Refere que este fenômeno se deve basicamente a não resolutividade do sistema de saúde. Com relação a este aspecto, outros dois sujeitos relatam que as mudanças ocorrem, mas de forma muito lenta. Andrade e Inojosa ${ }^{9}$ referem que o Programa Saúde da Família tem a capacidade de manter, entre comunidade e serviço público, a relação de compromisso e confiança mútuos para, assim, estabelecer a co-produção da vida e da saúde da comunidade.

A partir do relato da existência de mudanças, solicitamos que os sujeitos apontassem que fato na organização do trabalho contribui para que estas ocorressem. Assim, nas falas foram identificados três fatores. O primeiro fator considerou que a mudança ocorrida foi relacionada à organização do serviço de saúde local que com a presença de funcionários, programas, visitas domiciliares, reunião com a chefia, acompanhamento dos tratamentos, trabalho de prevenção, divisão de áreas e microáreas, união das equipes, e seu empenho criou oportunidade de planejar o trabalho. O segundo fator destacado relacionou as mudanças ao envolvimento da população com a tomada de decisão de procurar o serviço de saúde para enfrentar suas questões de saúde. O terceiro fator destacou a união das duas características: a organização do trabalho da equipe do Programa e a aceitação e busca da comunidade para as atividades propostas, o que proporcionou as mudanças na intervenção em saúde e na realidade local.

Para que possa existir melhoria na atenção a saúde, o comprometimento dos profissionais envolvidos com o SUS e com o PSF deve ser amplo, pois é necessário conhecer o outro em sua plenitude para construir um sistema de atenção apropriado ${ }^{7}$. Portanto, deve-se redimensionar o papel dos profissionais de saúde e as suas relações com a comunidade, permitindo uma mudança que desloque a relação de autoritarismo para uma relação que permita desenvolver uma atenção adequada à cultura popular e às especificidades desta. O autor, ao relacionar a participação popular neste processo, refere que não se traduz apenas na formação de conselhos de saúde, mas numa bandeira de luta dos setores, em que o sujeito pertencente às classes populares é um ator fundamental para o sistema saúde. Afirma que cabe, então, aos profissionais comprometidos com a consolidação do SUS romper com as políticas que excluíam o cidadão dos serviços públicos. 
Os sujeitos desta investigação mostraram reflexão sobre a determinação social do processo saúde-doença e que ainda não conseguem perceber a intervenção do serviço local como a possibilidade de modificar esta determinação, pois a prática sanitária local está voltada para atender a demanda de sinais, sintomas e queixas e assim intervir com diagnósticos previamente estabelecidos sem refletirem sobre as especificidades de cada sujeito e a determinação do quadro que este apresenta.

Conforme Andrade et $\mathrm{al}^{10}$, os profissionais do SUS precisam compreender que fazer saúde é atuar sobre a saúde e não somente sobre a doença. Fundamentados nesta premissa, as equipes de saúde da família poderão estar contribuindo para a formação da autonomia dos cidadãos sobre sua vida e saúde. Pelo que percebemos nesta investigação, este é um desafio essencial para as equipes de saúde da família e nos princípios do Programa Saúde da Família a integralidade se constitui na visão totalizadora do indivíduo e/ou da comunidade. Neste sentido deve-se considerar os aspectos sociais, culturais e econômicos da população e para enfrentar as questões com toda a sua complexidade é que se faz mister o trabalho multidisciplinar com discussões e elaboração de atenção integral ao indivíduo.

\section{CONSIDERAÇÕES FINAIS}

Nesta pesquisa percebemos que o processo de territorialização na U.S. ocorreu no momento da implantação do serviço e é continuamente construído com a introdução dos novos dados da realidade ali vivida. Porém, nem todos os profissionais, sujeitos da pesquisa, têm a clareza de que a contínua construção do processo de territorialização é a base da discussão e construção do modelo de assistência à saúde para aquela comunidade. Ao falarem sobre o processo de territorialização, os entrevistados destacaram o delineamento do mapa e (re) divisão da área de abrangência, a contagem das famílias e dos indivíduos que freqüentam a Unidade de Saúde, desconsiderando ou não tendo a clareza sobre os dados referentes ao contexto econômico, social, cultural e epidemiológico local. Ao contrário, percebemos que nas falas há uma valorização dos dados quantitativos do processo como se estes dessem conta de representar o processo saúdedoença daquela comunidade.

Quanto ao modelo de assistência prestado pela equipe, pudemos perceber que este ainda tem como foco a demanda espontânea no serviço. No entanto, relataram que utilizam a visita domiciliar e as ações dos agentes comunitários de saúde para prestar atendimento a pacientes em seus domicílios. Para tal, destacam os de maior prioridade, ou seja, portadores de algum adoecimento ou com riscos à saúde identificados. Dentre estes, estão os problemas mais identificados nas áreas de atuação dos profissionais, como: doenças crônicas (diabetes e hipertensão), drogadição e alcoolismo, entre outros.

Ao relacionarem as mudanças no processo desde a implantação do serviço, os sujeitos destacaram aquelas que se relacionam ao serviço e poucas foram relacionadas com a realidade vivida na comunidade, sendo a higiene pessoal e residencial a mudança mais significativa desde a implantação do serviço local. Enfatizaram ainda que o processo de mudança é muito lento.

Entretanto, verificamos que a comunidade não discute com a equipe as ações necessárias para a mudança no quadro de saúde e doença que apresentam. Sobre esta questão destacamos a importância da participação dos usuários no Conselho Local de Saúde, que é o espaço democrático garantido pelos princípios do SUS.

Outro ponto que foi caracterizado pelos entrevistados e que tem afetado o serviço é a falta de profissionais e o pequeno tempo de permanência dos mesmos no Programa. Isto nos remete a outra reflexão sobre a qualificação de profissionais para desenvolver um trabalho que tem como enfoque a promoção à saúde centrado na abordagem com a família, o que são propostas inovadoras na prática sanitária local. Logo, seria necessária uma maior 
vinculação destes profissionais para a construção e consolidação do novo modelo de atenção à saúde.

Ao concluirmos, afirmamos que no desenvolvimento deste estudo foi possível atingir os objetivos propostos, pois a coleta de dados por meio de entrevistas e da observação estruturada nos permitiu investigar e compreender o processo de territorialização e a implantação da assistência a saúde na U.S.; que ao desenvolvermos o estudo percebemos que estamos longe de conseguirmos desenvolver o modelo de atenção à saúde proposto pelo Programa Saúde da Família. Para tal, necessitaremos de muitas investigações, que como esta nos façam refletir sobre as mais diversas realidades de saúde e as ações que os serviços estão desenvolvendo.

\section{REFERÊNCIAS}

1 Ministério da Saúde (BR). Manual de enfermagem. Instituto para o desenvolvimento da saúde. Brasília: USP; 2001.

2 Rouquayrol MZ, Almeida Filho N. Epidemiologia e saúde. 6. ed. Rio de Janeiro: MEDSI; 2003.
3 Polit DF, Hungler BP. Fundamentos de pesquisa em enfermagem. 3. ed. Porto Alegre: Artes Médicas; 1995.

4 Minayo MCS. O desafio do conhecimento: pesquisa qualitativa em saúde. 4.ed. São Paulo-Rio de Janeiro: Hucitec-Abrasco; 1996.

5 Souza LE. A utilização da microlocalização de problemas na vigilância à saúde em distritos sanitários. A vigilância à saúde no distrito sanitário. Brasília: OPAS/OMS; 1992. p. $71-88$.

6 Trad LAB, et al. Estudo etnográfico da satisfação do usuário do programa saúde da família (PSF) na Bahia. Ciência e saúde coletiva 2002; 7(3): 581-9.

7 Oliveira FJA. Participação popular em saúde: as dimensões da cultura. Saúde em Debate 1996; 52: 67-73.

8 Ministério da Saúde (BR). Avaliação normativa do programa saúde da família no Brasil: monitoramento da implantação e funcionamento das equipes de saúde da família: 2001-2002. Brasília; 2004.

9 Andrade LOM, Inojosa RM. Saúde da Família, violência e Cultura da Paz. Rev Bras de Saúde da Família 2004; 5(ne): 55-61.

10 Andrade LOM, et al. Organização da atenção básica e estratégia saúde da família no município de Sobral Ceará - Brasil: principais avanços e desafios na perspectiva de produzir mudanças positivas na saúde. Experiências e desafios da atenção básica e saúde familiar: caso Brasil. Brasília: Organização PanAmericana de Saúde; 2004. p. 143-79. 